\title{
A RESULT IN THE SPIRIT OF HERSTEIN THEOREM
}

\author{
Maja Fošner, Benjamin Marcen and Joso Vukman \\ University of Maribor, Slovenia
}

\begin{abstract}
A classical result of Herstein asserts that any Jordan derivation on a prime ring of characteristic different from two is a derivation. It is our aim in this paper to prove the following result, which is in the spirit of Herstein's theorem. Let $n \geq 3$ be some fixed integer, let $R$ be a prime ring with $\operatorname{char}(R)>4 n-8$ and let $D: R \rightarrow R$ be an additive mapping satisfying either the relation $D\left(x^{n}\right)=D\left(x^{n-1}\right) x+x^{n-1} D(x)$ or the relation $D\left(x^{n}\right)=D(x) x^{n-1}+x D\left(x^{n-1}\right)$ for all $x \in R$. In both cases $D$ is a derivation.
\end{abstract}

This research has been motivated by the recent work of Vukman [15]. Throughout, $R$ will represent an associative ring with center $Z(R)$. As usual we write $[x, y]$ for $x y-y x$. Given an integer $n \geq 2$, a ring $R$ is said to be $n$-torsion free, if for $x \in R, n x=0$ implies $x=0$. Recall that a ring $R$ is prime if for $a, b \in R, a R b=(0)$ implies that either $a=0$ or $b=0$ and is semiprime in case $a R a=(0)$ implies $a=0$. We denote by $\operatorname{char}(R)$ the characteristics of a prime ring $R$. An additive mapping $D: R \rightarrow R$, where $R$ is an arbitrary ring, is called a derivation if $D(x y)=D(x) y+x D(y)$ holds for all pairs $x, y \in R$ and is called a Jordan derivation in case $D\left(x^{2}\right)=$ $D(x) x+x D(x)$ is fulfilled for all $x \in R$. A derivation $D$ is inner in case there exists $a \in R$, such that $D(x)=[x, a]$ holds for all $x \in R$. Every derivation is a Jordan derivation. The converse is in general not true. A classical result of Herstein ([12]) asserts that any Jordan derivation on a 2torsion free prime ring is a derivation. A brief proof of Herstein's result can be found in [7]. Cusack ([10]) generalized Herstein theorem to 2-torsion free semiprime rings (see also [3] for an alternative proof). Let us point out that Beidar, Brešar, Chebotar and Martindale ([1]) have considerably generalized

2010 Mathematics Subject Classification. 16W10, 39B05.

Key words and phrases. Prime ring, semiprime ring, derivation, Jordan derivation, functional identity. 
Herstein theorem. A generalization of Herstein theorem can be found also in [8].

Motivated by the work of Brešar [4] and Vukman [15] has recently proved the following result.

THEOREM 1. Let $R$ be a 2-torsion free semiprime ring and let $D: R \rightarrow R$ be an additive mapping. Suppose that either

$$
D(x y x)=D(x y) x+x y D(x)
$$

or

$$
D(x y x)=D(x) y x+x D(y x)
$$

holds for all pairs $x, y \in R$. In both cases $D$ is a derivation.

Putting $y=x^{n-2}$ in (1) and (2) we obtain

$$
D\left(x^{n}\right)=D\left(x^{n-1}\right) x+x^{n-1} D(x)
$$

and

$$
D\left(x^{n}\right)=D(x) x^{n-1}+x D\left(x^{n-1}\right) .
$$

It is our aim in this paper to prove the following result, which is related to the equations above.

THEOREM 2. Let $n \geq 3$ be some fixed integer, let $R$ be a prime ring with $\operatorname{char}(R)>4 n-8$ and let $D: R \rightarrow R$ be an additive mapping satisfying either the relation

$$
D\left(x^{n}\right)=D\left(x^{n-1}\right) x+x^{n-1} D(x)
$$

or the relation

$$
D\left(x^{n}\right)=D(x) x^{n-1}+x D\left(x^{n-1}\right)
$$

for all $x \in R$. In both cases $D$ is a derivation.

In case $n=3$ Theorem 2 reduces to the result recently proved by M. Fošner and Peršin ([11]).

Theorem 2 is obtained as an application of the theory of functional identities (Beidar-Brešar-Chebotar theory). In particular, we shall use some ideas from the paper of Beidar and Fong [2] where bijective additive mappings preserving a fixed polynomial are characterized. The theory of functional identities considers set-theoretic mappings on rings that satisfy some identical relations. When treating such relations one usually concludes that the form of the mappings involved can be described, unless the ring is very special. We refer the reader to [5] for an introductory account on functional identities and to [6] for full treatment of this theory. For the proof of Theorem 2 we need Theorem 3 which might be of independent interest. 
Let $R$ be an algebra over a commutative ring $\phi$ and let

$$
p\left(x_{1}, x_{2}, x_{3}, \ldots, x_{n}\right)=\sum_{\pi \in S_{n}} x_{\pi(1)} x_{\pi(2)} x_{\pi(3)} \cdots x_{\pi(n)}
$$

be a fixed multilinear polynomial in noncommuting indeterminates $x_{i}$ over $\phi$. Here $S_{n}$ stands for the symmetric group of order n. Let $\mathcal{L}$ be a subset of $R$ closed under $p$, i.e. $p\left(\bar{x}_{n}\right) \in \mathcal{L}$ for all $x_{1}, x_{2}, x_{3}, \ldots, x_{n} \in \mathcal{L}$, where $\bar{x}_{n}=\left(x_{1}, x_{2}, x_{3}, \ldots, x_{n}\right)$. We shall consider a mapping $D: \mathcal{L} \rightarrow R$ satisfying

$$
\begin{aligned}
D\left(p\left(\bar{x}_{n}\right)\right)= & \sum_{\pi \in S_{n}} D\left(x_{\pi(1)} x_{\pi(2)} \cdots x_{\pi(n-1)}\right) x_{\pi(n)} \\
& +\sum_{\pi \in S_{n}} x_{\pi(1)} x_{\pi(2)} \cdots x_{\pi(n-1)} D\left(x_{\pi(n)}\right)
\end{aligned}
$$

for all $x_{1}, x_{2}, x_{3}, \ldots, x_{n} \in \mathcal{L}$. Let us mention that the idea of considering the expression $\left[p\left(\bar{x}_{n}\right), p\left(\bar{y}_{n}\right)\right]$ in its proof is taken from [2].

TheOREM 3. Let $\mathcal{L}$ be a $2 n$-free Lie subring of $R$ closed under $p$. If $D: \mathcal{L} \rightarrow R$ is an additive mapping satisfying (8), then $D$ is a derivation.

Proof. For any $a \in R$ and $\bar{x}_{n} \in \mathcal{L}^{n}$ we have

$$
\begin{aligned}
{\left[p\left(\bar{x}_{n}\right), a\right]=} & p\left(\left[x_{1}, a\right], x_{2}, x_{3}, \ldots, x_{n}\right)+p\left(x_{1},\left[x_{2}, a\right], x_{3}, \ldots, x_{n}\right)+\cdots \\
& +p\left(x_{1}, x_{2}, \ldots,\left[x_{n-1}, a\right], x_{n}\right)+p\left(x_{1}, x_{2}, \ldots, x_{n-1},\left[x_{n}, a\right]\right),
\end{aligned}
$$

and therefore

$$
\begin{aligned}
D\left[p\left(\bar{x}_{n}\right), a\right]= & \sum_{\pi \in S_{n}} D\left[x_{\pi(1)} x_{\pi(2)} \cdots x_{\pi(n-1)}, a\right] x_{\pi(n)} \\
& +\sum_{\pi \in S_{n}} D\left(x_{\pi(1)} x_{\pi(2)} \cdots x_{\pi(n-1)}\right)\left[x_{\pi(n)}, a\right] \\
& +\sum_{\pi \in S_{n}}\left[x_{\pi(1)} x_{\pi(2)} \cdots x_{\pi(n-1)}, a\right] D\left(x_{\pi(n)}\right) \\
& +\sum_{\pi \in S_{n}} x_{\pi(1)} x_{\pi(2)} \cdots x_{\pi(n-1)} D\left[x_{\pi(n)}, a\right] .
\end{aligned}
$$

In particular

$$
\begin{aligned}
D\left[p\left(\bar{x}_{n}\right), p\left(\bar{y}_{n}\right)\right]= & \sum_{\pi \in S_{n}} D\left[x_{\pi(1)} x_{\pi(2)} \cdots x_{\pi(n-1)}, p\left(\bar{y}_{n}\right)\right] x_{\pi(n)} \\
& +\sum_{\pi \in S_{n}} D\left(x_{\pi(1)} x_{\pi(2)} \cdots x_{\pi(n-1)}\right)\left[x_{\pi(n)}, p\left(\bar{y}_{n}\right)\right] \\
& +\sum_{\pi \in S_{n}}\left[x_{\pi(1)} x_{\pi(2)} \cdots x_{\pi(n-1)}, p\left(\bar{y}_{n}\right)\right] D\left(x_{\pi(n)}\right) \\
& +\sum_{\pi \in S_{n}} x_{\pi(1)} x_{\pi(2)} \cdots x_{\pi(n-1)} D\left[x_{\pi(n)}, p\left(\bar{y}_{n}\right)\right]
\end{aligned}
$$


Using

$$
\begin{aligned}
& D\left[x_{\pi(1)} x_{\pi(2)} \cdots x_{\pi(n-1)}, p\left(\bar{y}_{n}\right)\right]=-D\left[p\left(\bar{y}_{n}\right), x_{\pi(1)} x_{\pi(2)} \cdots x_{\pi(n-1)}\right] \\
& =\sum_{\sigma \in S_{n}} D\left[x_{\pi(1)} x_{\pi(2)} \cdots x_{\pi(n-1)}, y_{\sigma(1)} y_{\sigma(2)} \cdots y_{\sigma(n-1)}\right] y_{\sigma(n)} \\
& \quad+\sum_{\sigma \in S_{n}} D\left(y_{\sigma(1)} y_{\sigma(2)} \cdots y_{\sigma(n-1)}\right)\left[x_{\pi(1)} x_{\pi(2)} \cdots x_{\pi(n-1)}, y_{\sigma(n)}\right] \\
& \quad+\sum_{\sigma \in S_{n}}\left[x_{\pi(1)} x_{\pi(2)} \cdots x_{\pi(n-1)}, y_{\sigma(1)} y_{\sigma(2)} \cdots y_{\sigma(n-1)}\right] D\left(y_{\sigma(n)}\right) \\
& \quad+\sum_{\sigma \in S_{n}} y_{\sigma(1)} y_{\sigma(2)} \cdots y_{\sigma(n-1)} D\left[x_{\pi(1)} x_{\pi(2)} \cdots x_{\pi(n-1)}, y_{\sigma(n)}\right]
\end{aligned}
$$

and

$$
\begin{aligned}
& D\left[x_{\pi(n)}, p\left(\bar{y}_{n}\right)\right]=-D\left[p\left(\bar{y}_{n}\right), x_{\pi(n)}\right] \\
& =\sum_{\sigma \in S_{n}} D\left[x_{\pi(n)}, y_{\sigma(1)} y_{\sigma(2)} \cdots y_{\sigma(n-1)}\right] y_{\sigma(n)} \\
& \quad+\sum_{\sigma \in S_{n}} D\left(y_{\sigma(1)} y_{\sigma(2)} \cdots y_{\sigma(n-1)}\right)\left[x_{\pi(n)}, y_{\sigma(n)}\right] \\
& \quad+\sum_{\sigma \in S_{n}}\left[x_{\pi(n)}, y_{\sigma(1)} y_{\sigma(2)} \cdots y_{\sigma(n-1)}\right] D\left(y_{\sigma(n)}\right. \\
& \quad+\sum_{\sigma \in S_{n}} y_{\sigma(1)} y_{\sigma(2)} \cdots y_{\sigma(n-1)} D\left[x_{\pi(n)}, y_{\sigma(n)}\right]
\end{aligned}
$$

in (10) we have

(13)

$$
\begin{aligned}
D & {\left[p\left(\bar{x}_{n}\right), p\left(\bar{y}_{n}\right)\right] } \\
= & \sum_{\pi \in S_{n}} \sum_{\sigma \in S_{n}} D\left[x_{\pi(1)} x_{\pi(2)} \cdots x_{\pi(n-1)}, y_{\sigma(1)} y_{\sigma(2)} \cdots y_{\sigma(n-1)}\right] y_{\sigma(n)} x_{\pi(n)} \\
& +\sum_{\pi \in S_{n}} \sum_{\sigma \in S_{n}} D\left(y_{\sigma(1)} y_{\sigma(2)} \cdots y_{\sigma(n-1)}\right)\left[x_{\pi(1)} x_{\pi(2)} \cdots x_{\pi(n-1)}, y_{\sigma(n)}\right] x_{\pi(n)} \\
& +\sum_{\pi \in S_{n}} \sum_{\sigma \in S_{n}}\left[x_{\pi(1)} x_{\pi(2)} \cdots x_{\pi(n-1)}, y_{\sigma(1)} y_{\sigma(2)} \cdots y_{\sigma(n-1)}\right] D\left(y_{\sigma(n)}\right) x_{\pi(n)} \\
& +\sum_{\pi \in S_{n}} \sum_{\sigma \in S_{n}} y_{\sigma(1)} y_{\sigma(2)} \cdots y_{\sigma(n-1)} D\left[x_{\pi(1)} x_{\pi(2)} \cdots x_{\pi(n-1)}, y_{\sigma(n)}\right] x_{\pi(n)} \\
& +\sum_{\pi \in S_{n}} \sum_{\sigma \in S_{n}} D\left(x_{\pi(1)} x_{\pi(2)} \cdots x_{\pi(n-1)}\right)\left[x_{\pi(n)}, y_{\sigma(1)} y_{\sigma(2)} \cdots y_{\sigma(n-1)} y_{\sigma(n)}\right]
\end{aligned}
$$


$+\sum_{\pi \in S_{n}} \sum_{\sigma \in S_{n}}\left[x_{\pi(1)} x_{\pi(2)} \cdots x_{\pi(n-1)}, y_{\sigma(1)} y_{\sigma(2)} \cdots y_{\sigma(n-1)} y_{\sigma(n)}\right] D\left(x_{\pi(n)}\right)$

$+\sum_{\pi \in S_{n}} \sum_{\sigma \in S_{n}} x_{\pi(1)} x_{\pi(2)} \cdots x_{\pi(n-1)} D\left[x_{\pi(n)}, y_{\sigma(1)} y_{\sigma(2)} \cdots y_{\sigma(n-1)}\right] y_{\sigma(n)}$

$+\sum_{\pi \in S_{n}} \sum_{\sigma \in S_{n}} x_{\pi(1)} x_{\pi(2)} \cdots x_{\pi(n-1)} D\left(y_{\sigma(1)} y_{\sigma(2)} \cdots y_{\sigma(n-1)}\right)\left[x_{\pi(n)}, y_{\sigma(n)}\right]$

$+\sum_{\pi \in S_{n}} \sum_{\sigma \in S_{n}} x_{\pi(1)} x_{\pi(2)} \cdots x_{\pi(n-1)}\left[x_{\pi(n)}, y_{\sigma(1)} y_{\sigma(2)} \cdots y_{\sigma(n-1)}\right] D\left(y_{\sigma(n)}\right)$

$+\sum_{\pi \in S_{n}} \sum_{\sigma \in S_{n}} x_{\pi(1)} x_{\pi(2)} \cdots x_{\pi(n-1)} y_{\sigma(1)} y_{\sigma(2)} \cdots y_{\sigma(n-1)} D\left[x_{\pi(n)}, y_{\sigma(n)}\right]$

If we replace the roles of $\pi$ and $\sigma$ we get

(14)

$D\left[p\left(\bar{x}_{n}\right), p\left(\bar{y}_{n}\right)\right]$

$=\sum_{\pi \in S_{n}} \sum_{\sigma \in S_{n}} D\left[x_{\pi(1)} x_{\pi(2)} \cdots x_{\pi(n-1)}, y_{\sigma(1)} y_{\sigma(2)} \cdots y_{\sigma(n-1)}\right] x_{\pi(n)} y_{\sigma(n)}$

$+\sum_{\pi \in S_{n}} \sum_{\sigma \in S_{n}} D\left(x_{\pi(1)} x_{\pi(2)} \cdots x_{\pi(n-1)}\right)\left[x_{\pi(n)}, y_{\sigma(1)} y_{\sigma(2)} \cdots y_{\sigma(n-1)}\right] y_{\sigma(n)}$

$+\sum_{\pi \in S_{n}} \sum_{\sigma \in S_{n}}\left[x_{\pi(1)} x_{\pi(2)} \cdots x_{\pi(n-1)}, y_{\sigma(1)} y_{\sigma(2)} \cdots y_{\sigma(n-1)}\right] D\left(x_{\pi(n)}\right) y_{\sigma(n)}$

$+\sum_{\pi \in S_{n}} \sum_{\sigma \in S_{n}} x_{\pi(1)} x_{\pi(2)} \cdots x_{\pi(n-1)} D\left[x_{\pi(n)}, y_{\sigma(1)} y_{\sigma(2)} \cdots y_{\sigma(n-1)}\right] y_{\sigma(n)}$

$+\sum_{\pi \in S_{n}} \sum_{\sigma \in S_{n}} D\left(y_{\sigma(1)} y_{\sigma(2)} \cdots y_{\sigma(n-1)}\right)\left[x_{\pi(1)} x_{\pi(2)} \cdots x_{\pi(n-1)} x_{\pi(n)}, y_{\sigma(n)}\right]$

$\left.+\sum_{\pi \in S_{n}} \sum_{\sigma \in S_{n}}\left[x_{\pi(1)} x_{\pi(2)} \cdots x_{\pi(n-1)} x_{\pi(n)}, y_{\sigma(1)} y_{\sigma(2)} \cdots y_{\sigma(n-1)}\right)\right] D\left(y_{\sigma(n)}\right)$

$+\sum_{\pi \in S_{n}} \sum_{\sigma \in S_{n}} y_{\sigma(1)} y_{\sigma(2)} \cdots y_{\sigma(n-1)} D\left[x_{\pi(1)} x_{\pi(2)} \cdots x_{\pi(n-1)}, y_{\sigma(n)}\right] x_{\pi(n)}$

$+\sum_{\pi \in S_{n}} \sum_{\sigma \in S_{n}} y_{\sigma(1)} y_{\sigma(2)} \cdots y_{\sigma(n-1)} D\left(x_{\pi(1)} x_{\pi(2)} \cdots x_{\pi(n-1)}\right)\left[x_{\pi(n)}, y_{\sigma(n)}\right]$

$+\sum_{\pi \in S_{n}} \sum_{\sigma \in S_{n}} y_{\sigma(1)} y_{\sigma(2)} \cdots y_{\sigma(n-1)}\left[x_{\pi(1)} x_{\pi(2)} \cdots x_{\pi(n-1)}, y_{\sigma(n)}\right] D\left(x_{\pi(n)}\right)$

$+\sum_{\pi \in S_{n}} \sum_{\sigma \in S_{n}} y_{\sigma(1)} y_{\sigma(2)} \cdots y_{\sigma(n-1)} x_{\pi(1)} x_{\pi(2)} \cdots x_{\pi(n-1)} D\left[x_{\pi(n)}, y_{\sigma(n)}\right]$. 
It follows from both (13) and (14) that

(15)

$$
\begin{aligned}
0 & \sum_{\pi \in S_{n}} \sum_{\sigma \in S_{n}}\left(D\left[x_{\pi(1)} x_{\pi(2)} \cdots x_{\pi(n-1)}, y_{\sigma(1)} y_{\sigma(2)} \cdots y_{\sigma(n-1)}\right]\right. \\
& +D\left(y_{\sigma(1)} y_{\sigma(2)} \cdots y_{\sigma(n-1)}\right) x_{\pi(1)} x_{\pi(2)} \cdots x_{\pi(n-1)} \\
& -D\left(x_{\pi(1)} x_{\pi(2)} \cdots x_{\pi(n-1)}\right) y_{\sigma(1)} y_{\sigma(2)} \cdots y_{\sigma(n-1)} \\
& -x_{\pi(1)} x_{\pi(2)} \cdots x_{\pi(n-1)} D\left(y_{\sigma(1)} y_{\sigma(2)} \cdots y_{\sigma(n-1)}\right) \\
& \left.+y_{\sigma(1)} y_{\sigma(2)} \cdots y_{\sigma(n-1)} D\left(x_{\pi(1)} x_{\pi(2)} \cdots x_{\pi(n-1)}\right)\right) y_{\sigma(n)} x_{\pi(n)} \\
& +\sum_{\pi \in S_{n}} \sum_{\sigma \in S_{n}}\left(x_{\pi(1)} x_{\pi(2)} \cdots x_{\pi(n-1)} D\left(y_{\sigma(1)} y_{\sigma(2)} \cdots y_{\sigma(n-1)}\right)\right. \\
& -D\left[x_{\pi(1)} x_{\pi(2)} \cdots x_{\pi(n-1)}, y_{\sigma(1)} y_{\sigma(2)} \cdots y_{\sigma(n-1)}\right] \\
& +D\left(x_{\pi(1)} x_{\pi(2)} \cdots x_{\pi(n-1)}\right) y_{\sigma(1)} y_{\sigma(2)} \cdots y_{\sigma(n-1)} \\
& -D\left(y_{\sigma(1)} y_{\sigma(2)} \cdots y_{\sigma(n-1)}\right) x_{\pi(1)} x_{\pi(2)} \cdots x_{\pi(n-1)} \\
& \left.-y_{\sigma(1)} y_{\sigma(2)} \cdots y_{\sigma(n-1)} D\left(x_{\pi(1)} x_{\pi(2)} \cdots x_{\pi(n-1)}\right)\right) x_{\pi(n)} y_{\sigma(n)} \\
& +\sum_{\pi \in S_{n}} \sum_{\sigma \in S_{n}} x_{\pi(1)} x_{\pi(2)} \cdots x_{\pi(n-1)}\left(y_{\sigma(1)} y_{\sigma(2)} \cdots y_{\sigma(n-1)} D\left(y_{\sigma(n)}\right) x_{\pi(n)}\right. \\
& -y_{\sigma(1)} y_{\sigma(2)} \cdots y_{\sigma(n-1)} x_{\pi(n)} D\left(y_{\sigma(n)}\right) \\
& +y_{\sigma(1)} y_{\sigma(2)} \cdots y_{\sigma(n-1)} D\left[x_{\pi(n)}, y_{\sigma(n)}\right] \\
& +y_{\sigma(1)} y_{\sigma(2)} \cdots y_{\sigma(n-1)} y_{\sigma(n)} D\left(x_{\pi(n)}\right) \\
& \left.-y_{\sigma(1)} y_{\sigma(2)} \cdots y_{\sigma(n-1)} D\left(x_{\pi(n)}\right) y_{\sigma(n)}\right) \\
& +\sum_{\pi \in S_{n}} \sum_{\sigma \in S_{n}} y_{\sigma(1)} y_{\sigma(2)} \cdots y_{\sigma(n-1)}\left(x_{\pi(1)} x_{\pi(2)} \cdots x_{\pi(n-1)} D\left(x_{\pi(n)}\right) y_{\sigma(n)}\right. \\
& -x_{\pi(1)} x_{\pi(2)} \cdots x_{\pi(n-1)} D\left(y_{\sigma(n)}\right) x_{\pi(n)} \\
& -x_{\pi(1)} x_{\pi(2)} \cdots x_{\pi(n-1)} y_{\sigma(n)} D\left(x_{\pi(n)}\right) \\
& -x_{\pi(1)} x_{\pi(2)} \cdots x_{\pi(n-1)} D\left[x_{\pi(n)}, y_{\sigma(n)}\right] \\
& \left.+x_{\pi(1)} x_{\pi(2)} \cdots x_{\pi(n-1)} x_{\pi(n)} D\left(y_{\sigma(n)}\right)\right) \\
& \cdots
\end{aligned}
$$

for all $x_{1}, x_{2}, \ldots, x_{n}, y_{1}, y_{2}, \ldots, y_{n} \in \mathcal{L}$. Now using the theory of functional identities we apply the definition of $n$-freeness of $\mathcal{L}$ so that there exist mappins $p_{1, j}: \mathcal{L}^{n} \rightarrow R, j=1, \ldots,(n+1)$ and $\lambda_{1}: \mathcal{L}^{n+1} \rightarrow C(\mathcal{L})$ such that the last equation can be rewritte as:

$$
\begin{aligned}
& \sum_{\pi \in S_{n}} \sum_{\sigma \in S_{n}}\left(y_{\sigma(1)} y_{\sigma(2)} \cdots y_{\sigma(n-1)} D\left(y_{\sigma(n)}\right) x_{\pi(n)}\right. \\
& \quad-y_{\sigma(1)} y_{\sigma(2)} \cdots y_{\sigma(n-1)} x_{\pi(n)} D\left(y_{\sigma(n)}\right) \\
& \quad+y_{\sigma(1)} y_{\sigma(2)} \cdots y_{\sigma(n-1)} D\left[x_{\pi(n)}, y_{\sigma(n)}\right] \\
& \quad+y_{\sigma(1)} y_{\sigma(2)} \cdots y_{\sigma(n-1)} y_{\sigma(n)} D\left(x_{\pi(n)}\right)
\end{aligned}
$$




$$
\begin{aligned}
& \left.\quad-y_{\sigma(1)} y_{\sigma(2)} \cdots y_{\sigma(n-1)} D\left(x_{\pi(n)}\right) y_{\sigma(n)}\right) \\
= & \sum_{j=1}^{n+1} p_{1, j}\left(\bar{x}_{n+1}^{j}\right) x_{j}+\lambda_{1}\left(\bar{x}_{n+1}\right)
\end{aligned}
$$

for all $\bar{x}_{n+1} \in \mathcal{L}^{n+1}$. After a finite number of steps using the same arguments as above we arrive at

$$
D(x) y-D(y) x-y D(x)-D([x, y])+x D(y)=f(x) y+g(y) x+\mu(x, y)
$$

for all $x, y \in \mathcal{L}$, where $f, g: \mathcal{L} \rightarrow R$ and $\mu: \mathcal{L}^{2} \rightarrow C(\mathcal{L})$. Hence

$$
\begin{aligned}
D([x, y])= & D(x) y-D(y) x-y D(x)+x D(y) \\
& -f(x) y-g(y) x-\mu(x, y) .
\end{aligned}
$$

If we replace the roles of denotations $x$ and $y$ in (17) and compare so obtained identities we arrive at $0=-f(x) y-g(y) x-\mu(x, y)-f(y) x-g(x) y-\mu(y, x)$. Now we have $-f(x)=g(x)$ for all $x \in \mathcal{L}$. We also obtain $\mu(x, y)+\mu(y, x)=0$ and $\mu(x, x)=0$ for all $x, y \in \mathcal{L}$. Setting $x^{2}$ instead of $x$ and $x=y$ in (17) it follows

$$
\begin{aligned}
0= & D\left(x^{2}\right) x-D(x) x^{2}-x D\left(x^{2}\right)+x^{2} D(x) \\
& -f\left(x^{2}\right) x+f(x) x^{2}-\mu\left(x^{2}, x\right)
\end{aligned}
$$

for all $x \in \mathcal{L}$. After a complete linearization of this identity we arrive at

$$
-D(x y)-D(y x)+x D(y)+y D(x)=h(x) y+k(y) x+\lambda_{h}(x, y)
$$

and also

$$
\begin{aligned}
D(x y) & +D(y x)-D(x) y-D(y) x-f(x y)-f(y x)+f(x) y+f(y) x \\
= & x l(y)+y m(x)+\lambda_{l}(x, y)
\end{aligned}
$$

for all $x, y \in \mathcal{L}$, where $h, k, l, m: \mathcal{L} \rightarrow R$ and $\lambda_{h}, \lambda_{l}: \mathcal{L}^{2} \rightarrow C(\mathcal{L})$. If we replace the roles of denotations $x$ and $y$ in (19) and compare so obtained identities we arrive at $0=h(x) y+k(y) x+\lambda_{h}(x, y)-h(y) x-k(x) y-\lambda_{h}(y, x)$. Now we have $h(x)=k(x)$ for all $x \in \mathcal{L}$. We also obtain $\lambda_{h}(x, y)=\lambda_{h}(y, x)$ for all $x, y \in \mathcal{L}$. Equation (19) can now be rewritten as

$$
-D(x y)-D(y x)+x D(y)+y D(x)=h(x) y+h(y) x+\lambda_{h}(x, y) .
$$

Similar if we replace the roles of denotations $x$ and $y$ in (20) and compare so obtained identities we arrive at $0=x l(y)+y m(x)+\lambda_{l}(x, y)-y l(x)-$ $x m(y)-\lambda_{l}(y, x)$. Now we have $l(x)=m(x)$ for all $x \in \mathcal{L}$. We also obtain $\lambda_{l}(x, y)=\lambda_{l}(y, x)$ for all $x, y \in \mathcal{L}$. Equation $(20)$ can now be rewritten as

$$
\begin{aligned}
& D(x y)+D(y x)-D(x) y-D(y) x-f(x y)-f(y x)+f(x) y+f(y) x \\
& =x l(y)+y l(x)+\lambda_{l}(x, y) .
\end{aligned}
$$

If $y=x$ in (21) and (22) we obtain

$$
2 D\left(x^{2}\right)=2 x D(x)-2 h(x) x-\lambda_{h}(x, x)
$$


and also

$$
2 D\left(x^{2}\right)=2 D(x) x+2 f\left(x^{2}\right)-2 f(x) x+2 x l(x)+\lambda_{l}(x, x) .
$$

Putting (24) into (18) leads to

$$
\begin{aligned}
0= & x l(x) x-x D(x) x-x f\left(x^{2}\right)+x f(x) x \\
& -x^{2} l(x)+x^{2} D(x)-\mu\left(x^{2}, x\right) .
\end{aligned}
$$

After complete linearization of the last equation we get

$$
\begin{aligned}
0= & \sum_{\pi \in S_{n}} x_{\pi(1)} l\left(x_{\pi(2)}\right) x_{\pi(3)}-x_{\pi(1)} D\left(x_{\pi(2)}\right) x_{\pi(3)} \\
& -x_{\pi(1)} f\left(x_{\pi(2)} x_{\pi(3)}\right)+x_{\pi(1)} f\left(x_{\pi(2)}\right) x_{\pi(3)} \\
& -x_{\pi(1)} x_{\pi(2)} l\left(x_{\pi(3)}\right)+x_{\pi(1)} x_{\pi(2)} D\left(x_{\pi(3)}\right)-\mu\left(x_{\pi(1)} x_{\pi(2)}, x_{\pi(3)}\right) .
\end{aligned}
$$

Using the theory of functional identities we obtain

$$
\begin{aligned}
0= & l(x) y+l(y) x-D(x) y-D(y) x-f(x y)-f(y x) \\
& +f(x) y+f(y) x-x l(y)-y l(x)+x D(y)+y D(x)
\end{aligned}
$$

and $\mu\left(x^{2}, x\right)=0$. Setting $y=x$ into the last equation we get

$$
f\left(x^{2}\right)=l(x) x-D(x) x+f(x) x-x l(x)+x D(x) .
$$

Now putting last equation into (24) we obtain

$$
2 D\left(x^{2}\right)=2 x D(x)+2 l(x) x+\lambda_{l}(x, x) .
$$

Comparing (23) and (26) we obtain $0=-h(x) x-l(x) x-\lambda_{h}(x, x)-\lambda_{l}(x, x)$.

This yields $-h(x)=l(x)$ and $-\lambda_{h}(x, x)=\lambda_{l}(x, x)$.

From $(21)$ we get $D(y x)=-D(x y)+x D(y)+y D(x)-h(x) y-h(y) x-$ $\lambda_{h}(x, y)$. Using this relation in (17) we get

$$
\begin{aligned}
2 D(x y)= & D(x) y-D(y) x+2 x D(y)-f(x) y+f(y) x \\
& -h(x) y-h(y) x-\mu(x, y)-\lambda_{h}(x, y) .
\end{aligned}
$$

Setting $x^{n}$ instead of $x$ and $y=x^{2}$ into (17) we get

$$
\begin{aligned}
0= & D\left(x^{n}\right) x^{2}-D\left(x^{2}\right) x^{n}-x^{2} D\left(x^{n}\right)+x^{n} D\left(x^{2}\right) \\
& -f\left(x^{n}\right) x^{2}+f\left(x^{2}\right) x^{n}-\mu\left(x^{n}, x^{2}\right) .
\end{aligned}
$$

Putting (5) into the last equation leads to

$$
\begin{aligned}
0= & D\left(x^{n-1}\right) x^{3}+x^{n-1} D(x) x^{2}-D\left(x^{2}\right) x^{n}-x^{2} D\left(x^{n-1}\right) x \\
& -x^{n+1} D(x)+x^{n} D\left(x^{2}\right)-f\left(x^{n}\right) x^{2}+f\left(x^{2}\right) x^{n}-\mu\left(x^{n}, x^{2}\right) .
\end{aligned}
$$

Now putting (23) into (27) we obtain

$$
\begin{aligned}
0= & 2 D\left(x^{n-1}\right) x^{3}+2 x^{n-1} D(x) x^{2}-2 x D(x) x^{n}+2 h(x) x^{n+1} \\
& +\lambda_{h}(x, x) x^{n}-2 x^{2} D\left(x^{n-1}\right) x-2 x^{n+1} D(x)+2 x^{n+1} D(x) \\
& -2 x^{n} h(x) x-x^{n} \lambda_{h}(x, x)-2 f\left(x^{n}\right) x^{2}+2 f\left(x^{2}\right) x^{n}-2 \mu\left(x^{n}, x^{2}\right) .
\end{aligned}
$$


Last equation can now be rewritten as

$$
\begin{aligned}
0= & 2 D\left(x^{n-1}\right) x^{3}+2 x^{n-1} D(x) x^{2}-2 x D(x) x^{n}+2 h(x) x^{n+1} \\
& -2 x^{2} D\left(x^{n-1}\right) x-2 x^{n} h(x) x-2 f\left(x^{n}\right) x^{2}+2 f\left(x^{2}\right) x^{n}-2 \mu\left(x^{n}, x^{2}\right) .
\end{aligned}
$$

Putting (25) into the last equation we get

$$
\begin{aligned}
0= & 2 D\left(x^{n-1}\right) x^{3}+2 x^{n-1} D(x) x^{2}-2 x D(x) x^{n}+2 h(x) x^{n+1} \\
& -2 x^{2} D\left(x^{n-1}\right) x-2 x^{n} h(x) x-2 f\left(x^{n}\right) x^{2}-2 h(x) x^{n+1} \\
& -2 D(x) x^{n+1}+2 f(x) x^{n+1}+2 x h(x) x^{n}+2 x D(x) x^{n}-2 \mu\left(x^{n}, x^{2}\right) .
\end{aligned}
$$

Last equation can now be rewritten as

$$
\begin{aligned}
0= & 2 D\left(x^{n-1}\right) x^{3}+2 x^{n-1} D(x) x^{2} \\
& -2 x^{2} D\left(x^{n-1}\right) x-2 x^{n} h(x) x-2 f\left(x^{n}\right) x^{2} \\
& -2 D(x) x^{n+1}+2 f(x) x^{n+1}+2 x h(x) x^{n}-2 \mu\left(x^{n}, x^{2}\right) .
\end{aligned}
$$

Setting $y=x^{n-1}$ into (17) we get

$$
\begin{aligned}
0= & D(x) x^{n-1}-D\left(x^{n-1}\right) x-x^{n-1} D(x)+x D\left(x^{n-1}\right) \\
& -f(x) x^{n-1}+f\left(x^{n-1}\right) x-\mu\left(x, x^{n-1}\right) .
\end{aligned}
$$

Last equation can now be rewritten as

$$
\begin{aligned}
2 D\left(x^{n-1}\right) x= & 2 D(x) x^{n-1}-2 x^{n-1} D(x)+2 x D\left(x^{n-1}\right) \\
& -2 f(x) x^{n-1}+2 f\left(x^{n-1}\right) x-2 \mu\left(x, x^{n-1}\right)
\end{aligned}
$$

or

$$
\begin{aligned}
2 x D\left(x^{n-1}\right)= & 2 D\left(x^{n-1}\right) x-2 D(x) x^{n-1}+2 x^{n-1} D(x) \\
& +2 f(x) x^{n-1}-2 f\left(x^{n-1}\right) x+2 \mu\left(x, x^{n-1}\right) .
\end{aligned}
$$

Putting (29) and (30) into (28) leads to

$$
\begin{aligned}
0= & 2 D(x) x^{n+1}-2 x^{n-1} D(x) x^{2}+2 x D\left(x^{n-1}\right) x^{2} \\
& -2 f(x) x^{n+1}+2 f\left(x^{n-1}\right) x^{3}-2 \mu\left(x, x^{n-1}\right) x^{2}+2 x^{n-1} D(x) x^{2} \\
& -2 x^{2} D\left(x^{n-1}\right) x-2 x^{n} h(x) x-2 f\left(x^{n}\right) x^{2} \\
& -2 D(x) x^{n+1}+2 f(x) x^{n+1}+2 x h(x) x^{n}-2 \mu\left(x^{n}, x^{2}\right) .
\end{aligned}
$$

Last equation can now be rewritten as

$$
\begin{aligned}
0= & 2 f\left(x^{n-1}\right) x^{3}-4 \mu\left(x, x^{n-1}\right) x^{2}+2 x D(x) x^{n} \\
& -2 x^{n} D(x) x-2 x f(x) x^{n}+2 x f\left(x^{n-1}\right) x^{2}-2 x^{n} h(x) x \\
& -2 f\left(x^{n}\right) x^{2}+2 x h(x) x^{n}-2 \mu\left(x^{n}, x^{2}\right) .
\end{aligned}
$$


After complete linearization of the last equation, using the theory of functional identities and setting $x_{\pi(1)}, x_{\pi(2)}, \ldots, x_{\pi(n+2)}=x$, we obtain $\mu\left(x^{n}, x^{2}\right)=0$ and

$$
\begin{aligned}
0= & 2 f\left(x^{n-1}\right) x^{2}-4 \mu\left(x, x^{n-1}\right) x+2 x D(x) x^{n-1} \\
& -2 x^{n} D(x)-2 x f(x) x^{n-1}+2 x f\left(x^{n-1}\right) x-2 x^{n} h(x) \\
& -2 f\left(x^{n}\right) x+2 x h(x) x^{n-1} .
\end{aligned}
$$

Now using theory of functional identities one more time after complete linearization we get

$$
\begin{aligned}
\sum_{\pi \in S_{n}} & -2 x_{\pi(2)} x_{\pi(3)} \cdots x_{\pi(n)} D\left(x_{\pi(n+1)}\right)-2 x_{\pi(2)} x_{\pi(3)} \cdots x_{\pi(n)} h\left(x_{n+1}\right) \\
& =\sum_{i=2}^{n+1} t_{1, i}\left(\bar{x}_{n}^{i}\right) x_{i}+\lambda_{t}\left(\bar{x}_{n}\right) .
\end{aligned}
$$

Continuing with the same procedure as above we obtain

$$
-2 D(x)-2 h(x)=p x+\lambda_{p}(x)
$$

for all $x \in L$, where $p \in R$ and $\lambda_{p}(x): \mathcal{L} \rightarrow C(\mathcal{L})$. Using $2 h(x)=-2 D(x)-$ $p x-\lambda_{p}(x)$ in (23) we get

$$
2 D\left(x^{2}\right)=2 D(x) x+2 x D(x)+p x^{2}+\lambda_{p}(x) x-\lambda_{h}(x, x) .
$$

Similar (24) can now be rewritten as

$$
2 D\left(x^{2}\right)=2 D(x) x+2 f\left(x^{2}\right)-2 f(x) x+2 x D(x)+x p x+x \lambda_{p}(x)-\lambda_{h}(x, x) .
$$

Now comparing last two equations we get

$$
2 f\left(x^{2}\right)=2 f(x) x+p x^{2}-x p x .
$$

Setting $x^{4}$ for $x$ and $y=x$ into (17) leads to

$$
\begin{aligned}
0= & 2 D\left(x^{4}\right) x-2 D(x) x^{4}-2 x D\left(x^{4}\right)+2 x^{4} D(x) \\
& -2 f\left(x^{4}\right) x+2 f(x) x^{4}-2 \mu\left(x^{4}, x\right) .
\end{aligned}
$$

Now putting $x^{2}$ instead of $x$ into (31) and (32) and using so obtained equations into (33) we get

$$
0=2 x^{2} p x^{3}-x p x^{4}-x^{3} p x^{2}-2 \mu\left(x^{4}, x\right) .
$$

After complete linearization of the last equation and using the theory of functional identities leads to $\mu\left(x^{4}, x\right)=0$ and

$$
0=2 x p x^{3}-p x^{4}-x^{2} p x^{2} .
$$

Using the theory of functional identities one more time we get

$$
0=2 x p x-p x^{2}-x^{2} p .
$$


The last equation can now be rewritten as $0=[[p, x], x]$. Now using Posner's theorem [8] it follows that $[p, x]=0$ for all $x \in R$. From (32) we now get $f\left(x^{2}\right)=f(x) x$. Complete linearization of (31) gives us

$$
\begin{aligned}
2 D(x y)+2 D(y x)= & 2 D(x) y+2 D(y) x+2 x D(y)+2 y D(x) \\
& +p x y+p y x+\lambda_{p}(x) y+\lambda_{p}(y) x-\lambda_{h}(x, y)-\lambda_{h}(y, x) .
\end{aligned}
$$

Setting $y=x^{n-1}$ in the last equation we get

$$
\begin{aligned}
4 D\left(x^{n}\right)= & 2 D(x) x^{n-1}+2 D\left(x^{n-1}\right) x+2 x D\left(x^{n-1}\right) \\
& +2 x^{n-1} D(x)+2 p x^{n}+\lambda_{p}(x) x^{n-1}+\lambda_{p}\left(x^{n-1}\right) x \\
& -\lambda_{h}\left(x, x^{n-1}\right)-\lambda_{h}\left(x^{n-1}, x\right) .
\end{aligned}
$$

Now using relation $D\left(x^{n}\right)=D\left(x^{n-1}\right) x+x^{n-1} D(x)$ in (34) leads to

$$
\begin{aligned}
0= & -2 D\left(x^{n-1}\right) x-2 x^{n-1} D(x)+2 D(x) x^{n-1}+2 x D\left(x^{n-1}\right) \\
& +2 p x^{n}+\lambda_{p}(x) x^{n-1}+\lambda_{p}\left(x^{n-1}\right) x-\lambda_{h}\left(x, x^{n-1}\right)-\lambda_{h}\left(x^{n-1}, x\right) .
\end{aligned}
$$

Putting $y=x^{n-1}$ into (17) and considering that $f\left(x^{n}\right)=f(x) x^{n-1}$ we get

$$
0=2 D(x) x^{n-1}-2 D\left(x^{n-1}\right) x-2 x^{n-1} D(x)+2 x D\left(x^{n-1}\right)-2 \mu\left(x, x^{n-1}\right) .
$$

Comparing last two equations leads to

$$
\begin{aligned}
0= & 2 p x^{n}+\lambda_{p}(x) x^{n-1}+\lambda_{p}\left(x^{n-1}\right) x \\
& -\lambda_{h}\left(x, x^{n-1}\right)-\lambda_{h}\left(x^{n-1}, x\right)+2 \mu\left(x, x^{n-1}\right) .
\end{aligned}
$$

Complete linearization of the last equation leads to

$$
\begin{aligned}
0= & \sum_{\pi \in S_{n}} 2 p x_{\pi(1)} x_{\pi(2)} x_{\pi(3)} \cdots x_{\pi(n)}+\lambda_{p}\left(x_{\pi(1)}\right) x_{\pi(2)} x_{\pi(3)} \cdots x_{\pi(n)} \\
& +\lambda_{p}\left(x_{\pi(1)} x_{\pi(2)} x_{\pi(3)} \cdots x_{\pi(n-1)}\right) x_{\pi(n)}-\lambda_{h}\left(x_{\pi(1)}, x_{\pi(2)} x_{\pi(3)} \cdots x_{\pi(n)}\right) \\
& -\lambda_{h}\left(x_{\pi(1)} x_{\pi(2)} x_{\pi(3)} \cdots x_{\pi(n-1)}, x_{\pi(n)}\right)+2 \mu\left(x_{\pi(1)}, x_{\pi(2)} x_{\pi(3)} \cdots x_{\pi(n)}\right) .
\end{aligned}
$$

Using the theory of functional identities leads to $\mu\left(x, x^{n-1}\right)=\lambda_{h}\left(x, x^{n-1}\right)$ and

$$
\begin{aligned}
0= & \sum_{\pi \in S_{n}} 2 p x_{\pi(1)} x_{\pi(2)} x_{\pi(3)} \cdots x_{\pi(n-1)}+\lambda_{p}\left(x_{\pi(1)}\right) x_{\pi(2)} x_{\pi(3)} \cdots x_{\pi(n-1)} \\
& +\lambda_{p}\left(x_{\pi(1)} x_{\pi(2)} x_{\pi(3)} \cdots x_{\pi(n-1)}\right) .
\end{aligned}
$$

Furthermore leads to $\lambda_{p}\left(x^{n-1}\right)=0$ and

$$
0=\sum_{\pi \in S_{n}} 2 p x_{\pi(1)} x_{\pi(2)} x_{\pi(3)} \cdots x_{\pi(n-2)}+\lambda_{p}\left(x_{\pi(1)}\right) x_{\pi(2)} x_{\pi(3)} \cdots x_{\pi(n-2)} .
$$

After using theory of functional identities few more times we get

$$
0=2 p x+\lambda_{p}(x) .
$$


We can now conclude that $p=0$ and $\lambda_{p}(x)=0$. Equation (31) can now be rewritten as

$$
2 D\left(x^{2}\right)=2 D(x) x+2 x D(x)-\lambda_{h}(x, x) .
$$

Setting $x^{2}$ instead of $x$ in the above relation gives

$$
2 D\left(x^{4}\right)=2 D\left(x^{2}\right) x^{2}+2 x^{2} D\left(x^{2}\right)-\lambda_{h}\left(x^{2}, x^{2}\right)
$$

and the last equation together with (35) can now be rewritten as

$$
\begin{aligned}
2 D\left(x^{4}\right)= & 2 D(x) x^{3}+2 x D(x) x^{2}+2 x^{2} D(x) x+2 x^{3} D(x) \\
& -2 x^{2} \lambda_{h}(x, x)-\lambda_{h}\left(x^{2}, x^{2}\right) .
\end{aligned}
$$

After complete linearization of (35) and setting $y=x^{3}$ we get

$$
4 D\left(x^{4}\right)=2 D(x) x^{3}+2 D\left(x^{3}\right) x+2 x D\left(x^{3}\right)+2 x^{3} D(x)-2 \lambda_{h}\left(x, x^{3}\right) .
$$

Comparing the last two identities leads to

$$
\begin{aligned}
0= & 2 D(x) x^{3}+4 x D(x) x^{2}+4 x^{2} D(x) x+2 x^{3} D(x) \\
& -2 D\left(x^{3}\right) x-2 x D\left(x^{3}\right)-4 x^{2} \lambda_{h}(x, x)-2 \lambda_{h}\left(x^{2}, x^{2}\right)+2 \lambda_{h}\left(x, x^{3}\right) .
\end{aligned}
$$

After complete linearization of (35) and setting $y=x^{2}$ we get

$$
4 D\left(x^{3}\right)=2 D(x) x^{2}+2 D\left(x^{2}\right) x+2 x D\left(x^{2}\right)+2 x^{2} D(x)-2 \lambda_{h}\left(x, x^{2}\right) .
$$

Putting last equation in (36) and using (35) leads to

$$
0=-4 x^{2} \lambda_{h}(x, x)+4 x \lambda_{h}\left(x, x^{2}\right)-4 \lambda_{h}\left(x^{2}, x^{2}\right)+4 \lambda_{h}\left(x, x^{3}\right) .
$$

After complete linearization of the last equation and using the theory of functional identities leads to $\lambda_{h}\left(x^{2}, x^{2}\right)=\lambda_{h}\left(x, x^{3}\right)$ and $0=-4 x \lambda_{h}(x, x)+$ $4 \lambda_{h}\left(x, x^{2}\right)$. Using theory one more time we get $\lambda_{h}\left(x, x^{2}\right)=0$ and $\lambda_{h}(x, x)=0$. Equation (35) can now be rewritten as

$$
2 D\left(x^{2}\right)=2 D(x) x+2 x D(x) .
$$

Consequently from the last equation follows that $D$ is a Jordan derivation. By Herstein theorem $D$ is a derivation. Thereby the proof is completed.

We are now in the position to prove Theorem 2.

Proof of TheOrem 2. The complete linearization of (5) gives us (8). Assume first that $R$ is not a PI ring. According to Theorem $3 D$ is a derivation.

Now suppose that $R$ is a PI ring. It is well-known that in this case $R$ has a nonzero center (see [13]). Let $c$ be a nonzero central element. Pick any $x \in R$. Next, setting $x_{1}=x_{2}=\cdots=x_{n-1}=c$ and $x_{n}=c x^{2}$ in (8) we arrive at

$$
\begin{aligned}
n D\left(c^{n} x^{2}\right)= & D\left(c^{n-1}\right) c x^{2}+(n-1) D\left(c^{n-1} x^{2}\right) c \\
& +c^{n-1} D\left(c x^{2}\right)+(n-1) c^{n-1} x^{2} D(c)
\end{aligned}
$$


for all $x \in R$. Next, setting $x_{1}=x_{2}=c x$ and $x_{3}=\cdots=x_{n}=c$ in (8) we arrive at

$$
\begin{aligned}
n D\left(c^{n} x^{2}\right)= & 2 D\left(x c^{n-1}\right) c x+(n-2) D\left(c^{n-1} x^{2}\right) c \\
& +2 c^{n-1} x D(c x)+(n-2) c^{n-1} x^{2} D(c)
\end{aligned}
$$

for all $x \in R$. Comparing last two equations we get

$$
\begin{aligned}
0= & D\left(c^{n-1}\right) c x^{2}+D\left(c^{n-1} x^{2}\right) c+c^{n-1} D\left(c x^{2}\right) \\
& +c^{n-1} x^{2} D(c)-2 D\left(x c^{n-1}\right) c x-2 x c^{n-1} D(c x)
\end{aligned}
$$

for all $x \in R$. Next, setting $x_{1}=\cdots=x_{n-1}=c$ and $x_{n}=x^{2}$ in (8) we arrive at

$$
\begin{aligned}
n D\left(c^{n-1} x^{2}\right)= & D\left(c^{n-1}\right) x^{2}+(n-1) D\left(c^{n-2} x^{2}\right) c \\
& +c^{n-1} D\left(x^{2}\right)+(n-1) c^{n-2} x^{2} D(c)
\end{aligned}
$$

for all $x \in R$. Now comparing the last equation and (37) we get

$$
\begin{aligned}
0= & (n+1) D\left(c^{n-1}\right) c x^{2}+(n-1) D\left(c^{n-2} x^{2}\right) c+c^{n} D\left(x^{2}\right) \\
& +(2 n-1) c^{n-1} x^{2} D(c)+n c^{n-1} D\left(c x^{2}\right) \\
& -2 n D\left(x c^{n-1}\right) c x-2 n x c^{n-1} D(c x)
\end{aligned}
$$

for all $x \in R$. Substituting $x$ for $c$ in last relation and using the relations $D\left(c^{n}\right)=D\left(c^{n-1}\right) c+c^{n-1} D(c)$ we get

$$
n D\left(c^{3}\right)=(2-n) c^{2} D(c)+(2 n-1) c D\left(c^{2}\right)
$$

for all $x \in R$. Next, setting $x_{1}=x, x_{2}=c x$ and $x_{3}=\cdots=x_{n}=c$ in (8) we arrive at

$$
\begin{aligned}
n D\left(c^{n-1} x^{2}\right)= & D\left(c^{n-1} x\right) x+D\left(c^{n-2} x\right) c x+(n-2) D\left(x^{2} c^{n-2}\right) c \\
& +x c^{n-1} D(x)+x c^{n-2} D(c x)+(n-2) c^{n-2} x^{2} D(c)
\end{aligned}
$$

for all $x \in R$. Setting $x_{1}=x, x_{2}=x$ and $x_{3}=\cdots=x_{n}=c$ in (8) we arrive at

$$
\begin{aligned}
n D\left(c^{n-2} x^{2}\right)= & 2 D\left(c^{n-2} x\right) x+(n-2) D\left(x^{2} c^{n-3}\right) c \\
& +2 x c^{n-2} D(x)+(n-2) c^{n-3} x^{2} D(c)
\end{aligned}
$$

for all $x \in R$. Now comparing (38) and (40) we get

$$
\begin{aligned}
0= & D\left(c^{n-1}\right) x^{2}+D\left(c^{n-2} x^{2}\right) c+c^{n-1} D\left(x^{2}\right) \\
& +c^{n-2} x^{2} D(c)-D\left(x c^{n-1}\right) x-D\left(c^{n-2} x\right) c x-x c^{n-1} D(x)-x c^{n-2} D(c x)
\end{aligned}
$$

for all $x \in R$. Comparing the last two equations we get

$$
\begin{aligned}
0= & n D\left(c^{n-1}\right) x^{2}+(2-n) D\left(c^{n-2} x\right) c x+(2-n) x c^{n-1} D(x) \\
& +(n-2) D\left(x^{2} c^{n-3}\right) c^{2}+(2 n-2) c^{n-2} x^{2} D(c) \\
& +n c^{n-1} D\left(x^{2}\right)-n D\left(x c^{n-1}\right) x-n x c^{n-2} D(c x)
\end{aligned}
$$


for all $x \in R$. Setting $x_{1}=x$ and $x_{2}=\cdots=x_{n}=c$ in (8) we arrive at

$$
\begin{aligned}
(n-1) D\left(c^{n-2} x\right) c= & n D\left(c^{n-1} x\right)-D\left(c^{n-1}\right) x-c^{n-1} D(x) \\
& -(n-1) x c^{n-2} D(c)
\end{aligned}
$$

for all $x \in R$. Comparing the last two equations we get

$$
\begin{aligned}
0= & (n-1) D\left(c^{n-1}\right) x^{2}+(3-2 n) D\left(c^{n-2} x\right) c x \\
& +(2-n) x c^{n-1} D(x)+(n-2) D\left(x^{2} c^{n-3}\right) c^{2}+(2 n-2) c^{n-2} x^{2} D(c) \\
& +n c^{n-1} D\left(x^{2}\right)-c^{n-1} D(x) x-(n-1) x c^{n-2} D(c) x-n x c^{n-2} D(c x)
\end{aligned}
$$

for all $x \in R$. Setting $c x$ instead of $x$ in the last equation we get

$$
\begin{aligned}
0= & (n-1) D\left(c^{n-1}\right) c^{2} x^{2}+(3-2 n) D\left(c^{n-1} x\right) c^{2} x \\
& +(2-n) x c^{n} D(c x)+(n-2) D\left(x^{2} c^{n-1}\right) c^{2}+(2 n-2) c^{n} x^{2} D(c) \\
& +n c^{n-1} D\left(c^{2} x^{2}\right)-c^{n} D(c x) x-(n-1) x c^{n} D(c) x-n x c^{n-1} D\left(c^{2} x\right)
\end{aligned}
$$

for all $x \in R$. Multiplying (41) by $c^{2}$ we obtain

$$
\begin{aligned}
0= & (n-1) D\left(c^{n-1}\right) c^{2} x^{2}+(3-2 n) D\left(c^{n-2} x\right) c^{3} x \\
& +(2-n) x c^{n+1} D(x)+(n-2) D\left(x^{2} c^{n-3}\right) c^{4}+(2 n-2) c^{n} x^{2} D(c) \\
& +n c^{n+1} D\left(x^{2}\right)-c^{n+1} D(x) x-(n-1) x c^{n} D(c) x-n x c^{n} D(c x)
\end{aligned}
$$

for all $x \in R$. Comparing (42) and (43) leads to

$$
\begin{aligned}
0= & (3-2 n) D\left(c^{n-1} x\right) c^{2} x+2 x c^{n} D(c x) \\
& +(n-2) D\left(x^{2} c^{n-1}\right) c^{2}+n c^{n-1} D\left(x^{2} c^{2}\right) \\
& -c^{n} D(c x) x-n x c^{n-1} D\left(c^{2} x\right) \\
& -(3-2 n) D\left(c^{n-2} x\right) c^{3} x-(2-n) x c^{n+1} D(x) \\
& -(n-2) D\left(x^{2} c^{n-3}\right) c^{4}-n c^{n+1} D\left(x^{2}\right)+c^{n+1} D(x) x .
\end{aligned}
$$

Setting $c$ instead of $x$ in (44) leads to

$$
\begin{aligned}
(2-n) D\left(c^{n+1}\right) c^{2}= & (-n+2) D\left(c^{n-1}\right) c^{4} \\
& +(2-3 n) c^{n+1} D\left(c^{2}\right)+n c^{n-1} D\left(c^{4}\right) .
\end{aligned}
$$

Setting $x_{1}=c$ and $x_{2}=\cdots=x_{n}=x$ in (8) we arrive at

$$
\begin{aligned}
n D\left(c x^{n-1}\right)= & D\left(x^{n-1}\right) c+(n-1) D\left(c x^{n-2}\right) x \\
& +x^{n-1} D(c)+(n-1) c x^{n-2} D(x)
\end{aligned}
$$

for all $x \in R$. Setting $x_{1}=x^{2}, x_{2}=c$ and $x_{3}=\cdots=x_{n}=x$ in (8) we arrive at

$$
\begin{aligned}
n D\left(c x^{n}\right)= & D\left(x^{n-2} c\right) x^{2}+D\left(x^{n}\right) c+(n-2) D\left(c x^{n-1}\right) x \\
& +x^{n-2} c D\left(x^{2}\right)+x^{n} D(c)+(n-2) c x^{n-1} D(x)
\end{aligned}
$$


for all $x \in R$. Setting $x_{1}=c x$ and $x_{2}=\cdots=x_{n}=x$ in (8) we arrive at

$$
\begin{aligned}
n D\left(c x^{n}\right)= & D\left(x^{n-1}\right) c x+(n-1) D\left(c x^{n-1}\right) x \\
& +x^{n-1} D(c x)+(n-1) c x^{n-1} D(x)
\end{aligned}
$$

for all $x \in R$. Comparing (46) and (47) we get

$$
\begin{aligned}
0= & D\left(c x^{n-2}\right) x^{2}+D\left(x^{n}\right) c-D\left(c x^{n-1}\right) x-D\left(x^{n-1}\right) c x \\
& +x^{n-2} c D\left(x^{2}\right)+x^{n} D(c)-c x^{n-1} D(x)-x^{n-1} D(c x) .
\end{aligned}
$$

Hence we get

$$
0=D\left(c x^{n-2}\right) x^{2}-D\left(c x^{n-1}\right) x+x^{n-2} c D\left(x^{2}\right)+x^{n} D(c)-x^{n-1} D(c x) .
$$

Putting (45) into last equation we get

$$
\begin{aligned}
0= & D\left(c x^{n-2}\right) x^{2}+n c x^{n-2} D\left(x^{2}\right)+n x^{n} D(c)-n x^{n-1} D(c x) \\
& -D\left(x^{n-1}\right) c x-x^{n-1} D(c) x-(n-1) c x^{n-2} D(x) x .
\end{aligned}
$$

Setting $c x$ instead of $x$ into (45) we get

$$
\begin{aligned}
n D\left(c^{n} x^{n-1}\right)= & D\left(c^{n-1} x^{n-1}\right) c+(n-1) D\left(c^{n-1} x^{n-2}\right) c x \\
& +c^{n-1} x^{n-1} D(c)+(n-1) c^{n-1} x^{n-2} D(c x) .
\end{aligned}
$$

Complete linearization of (48) and setting $x_{1}=x$ and $x_{2}=\cdots=x_{n}=c$ gives us

$$
\begin{aligned}
0= & D\left(c^{n-1}\right) c x-D\left(x c^{n-2}\right) c^{2}+n c^{n-1} D(c x) \\
& -n x c^{n-2} D\left(c^{2}\right)+(2 n-1) c^{n-1} x D(c)-n c^{n-1} D(c) x-(n-1) c^{n} D(x) .
\end{aligned}
$$

Now putting (41) into last equation we get

$$
\begin{aligned}
0= & D\left(c^{n-1}\right) x-D\left(x c^{n-1}\right)+(2-n) c^{n-1} D(x)+(2 n-2) x c^{n-2} D(c) \\
& +(n-1) c^{n-2} D(c x)-(n-1) x c^{n-3} D\left(c^{2}\right)-(n-1) c^{n-2} D(c) x .
\end{aligned}
$$

Setting $c x$ instead of $x$ into last equation we get

$$
\begin{aligned}
0= & D\left(c^{n-1}\right) c x-D\left(x c^{n}\right)+(2-n) c^{n-1} D(c x)+(2 n-2) x c^{n-1} D(c) \\
& +(n-1) c^{n-2} D\left(c^{2} x\right)-(n-1) x c^{n-2} D\left(c^{2}\right)-(n-1) c^{n-1} D(c) x .
\end{aligned}
$$

Comparing the last two equations we get

$$
\begin{aligned}
0= & -D\left(c^{n-1} x\right) c+(2-n) c^{n} D(x)+(2 n-3) c^{n-1} D(c x) \\
& +D\left(c^{n} x\right)-(n-1) c^{n-2} D\left(c^{2} x\right) .
\end{aligned}
$$

Setting $c$ instead of $x$ into last equation we get

$$
\begin{aligned}
0= & -D\left(c^{n-1}\right) c^{2}+(1-n) c^{n} D(c)+(2 n-3) c^{n-1} D\left(c^{2}\right) \\
& +D\left(c^{n+1}\right)+(1-n) c^{n-2} D\left(c^{3}\right) .
\end{aligned}
$$

Comparing (51) and (39) leads to

$$
n D\left(c^{n+1}\right)=n D\left(c^{n-1}\right) c^{2}+(2 n-2) c^{n} D(c)+c^{n-1} D\left(c^{2}\right) .
$$


Setting $x_{1}=c x$ and $x_{2}=\cdots=x_{n}=c$ in (8) we arrive at

$$
\begin{aligned}
n D\left(x c^{n}\right)= & D\left(c^{n-1}\right) c x+(n-1) D\left(x c^{n-1}\right) c \\
& +c^{n-1} D(c x)+(n-1) x c^{n-1} D(c)
\end{aligned}
$$

for all $x \in R$. Putting (52) into (50) we get

$$
\begin{aligned}
0= & -D\left(c^{n-1} x\right) c+\left(2 n-n^{2}\right) c^{n} D(x)+\left(2 n^{2}-3 n+1\right) c^{n-1} D(c x) \\
& +D\left(c^{n-1}\right) c x+(n-1) c^{n-1} x D(c)+\left(n-n^{2}\right) c^{n-2} D\left(c^{2} x\right) .
\end{aligned}
$$

Comparing (50) and (53) leads to

$$
\begin{aligned}
0= & \left(n^{2}-3 n+2\right) c^{n} D(x)+\left(-2 n^{2}+5 n-4\right) c^{n-1} D(c x)+D\left(c^{n} x\right) \\
& +\left(n^{2}-2 n+1\right) c^{n-2} D\left(c^{2} x\right)-D\left(c^{n-1}\right) c x+(1-n) c^{n-1} x D(c) .
\end{aligned}
$$

Now comparing (49) and (54) leads to

$$
\begin{aligned}
n D\left(c^{2} x\right)= & (2-n) c^{2} D(x)+2(n-1) c D(c x) \\
& -x c D(c)+c D(c) x+x D\left(c^{2}\right) .
\end{aligned}
$$

Setting $c^{2}$ instead of $x$ into the last equation and considering that

$$
n D\left(c^{3}\right)=(2-n) c^{2} D(c)+(2 n-1) c D\left(c^{2}\right)
$$

leads to

$$
n^{2} D\left(c^{4}\right)=\left(-2 n^{2}+6 n-4\right) c^{3} D(c)+\left(3 n^{2}-3 n+2\right) c^{2} D\left(c^{2}\right) .
$$

Multiplying (55) by $c^{2}$ leads to

$$
\begin{aligned}
n c^{2} D\left(c^{2} x\right)= & (2-n) c^{4} D(x)+2(n-1) c^{3} D(c x) \\
& -x c^{3} D(c)+c^{3} D(c) x+x c^{2} D\left(c^{2}\right) .
\end{aligned}
$$

On the other hand setting $c^{2}$ instead of $c$ into the (55) leads to

$$
\begin{aligned}
n D\left(c^{4} x\right)= & (2-n) c^{4} D(x)+2(n-1) c^{2} D\left(c^{2} x\right) \\
& -x c^{2} D\left(c^{2}\right)+c^{2} D\left(c^{2}\right) x+x D\left(c^{4}\right) .
\end{aligned}
$$

Now comparing the last two equations leads to

$$
\begin{aligned}
-n D\left(c^{4} x\right)= & (-3 n+2) c^{2} D\left(c^{2} x\right)+(2 n-2) c^{3} D(c x) \\
& -x c^{3} D(c)+c^{3} D(c) x+2 c^{2} x D\left(c^{2}\right)-c^{2} D\left(c^{2}\right) x-x D\left(c^{4}\right) .
\end{aligned}
$$

Setting $c^{2} x$ instead $x$ into the (55) leads to

$$
\begin{aligned}
n^{2} D\left(c^{4} x\right)= & n(2-n) c^{2} D\left(c^{2} x\right)+2 n(n-1) c D\left(c^{3} x\right) \\
& -n c^{3} x D(c)+n c^{3} D(c) x+n c^{2} x D\left(c^{2}\right) .
\end{aligned}
$$


Comparing the last two equations leads to

$$
\begin{aligned}
0= & \left(3 n^{2}-8 n+4\right) c^{4} D(x)+\left(-4 n^{2}+8 n-4\right) c^{3} D(c x) \\
& +(n-2) x c^{3} D(c)+(-n+2) c^{3} D(c) x+2 c^{2} x D\left(c^{2}\right)-n c^{2} D\left(c^{2}\right) x \\
& -n x D\left(c^{4}\right)+(2-n) n c^{2} D\left(c^{2} x\right)+2(n-1) n c D\left(c^{3} x\right) .
\end{aligned}
$$

Setting $c x$ instead of $x$ into the (55) and using (57) leads to

$$
\begin{aligned}
0= & \left(4 n^{2}-12 n+8\right) c^{4} D(x)+\left(-8 n^{2}+20 n-12\right) c^{3} D(c x) \\
& -2 x c^{3} D(c)+2 c^{3} D(c) x+(n+2) c^{2} x D\left(c^{2}\right)-n c^{2} D\left(c^{2}\right) x \\
& -n x D\left(c^{4}\right)+(2 n-2)^{2} c^{2} D\left(c^{2} x\right) .
\end{aligned}
$$

Now putting (55) and (56) into the last equation leads to

$$
\begin{aligned}
\left(4 n^{2}-12 n+8\right) c^{3} D(c x)= & \left(4 n^{2}-12 n+8\right) c^{4} D(x) \\
& -2 n^{2} x c^{3} D(c)+\left(4 n^{2}-6 n+4\right) c^{3} D(c) x \\
& +\left(2 n^{2}-3 n+2\right) c^{2} x D\left(c^{2}\right)-n^{2} c^{2} D\left(c^{2}\right) x .
\end{aligned}
$$

Setting $c$ instead of $x$ into the last equation we get

$$
D\left(c^{2}\right)=2 c D(c) .
$$

Using (60) into the (39) and (56) we get

$$
\begin{aligned}
& D\left(c^{3}\right)=3 c^{2} D(c), \\
& D\left(c^{4}\right)=4 c^{3} D(c) .
\end{aligned}
$$

Now we can derive that

$$
D\left(c^{n}\right)=n c^{n-1} D(c) .
$$

Using (61) the (59) can be rewritten as

$$
2 D(c x)=2 c D(x)+x D(c)+D(c) x .
$$

Using last equation, (61) and (55) leads to

$$
D\left(c^{2} x\right)=c^{2} D(x)+x c D(c)+c D(c) x .
$$

Comparing the last two equations leads to

$$
2 D\left(c^{2} x\right)=2 c D(c x)+x c D(c)+c D(c) x .
$$

Now setting $x_{1}=x_{2}=\cdots=x_{n-1}=c x$ and $x_{n}=x$ in (8). Hence we obtain

$$
\begin{aligned}
n D\left(c^{n-1} x^{n}\right)= & D\left(c^{n-1} x^{n-1}\right) x+(n-1) D\left(c^{n-2} x^{n-1}\right) c x \\
& +c^{n-1} x^{n-1} D(x)+(n-1) c^{n-2} x^{n-1} D(c x) .
\end{aligned}
$$

Next, setting $x_{1}=x_{2}=\cdots=x_{n-1}=c$ and $x_{n}=x^{n}$ in (8) we arrive at

$$
\begin{aligned}
n D\left(c^{n-1} x^{n}\right)= & D\left(c^{n-1}\right) x^{n}+(n-1) D\left(c^{n-2} x^{n}\right) c \\
& +c^{n-1}\left(D\left(x^{n-1}\right) x+x^{n-1} D(x)\right)+(n-1) c^{n-2} x^{n} D(c)
\end{aligned}
$$


for all $x \in R$. Comparing both identities we get

$$
\begin{aligned}
0= & D\left(c^{n-1} x^{n-1}\right) x+(n-1) D\left(c^{n-2} x^{n-1}\right) c x \\
& +(n-1) c^{n-2} x^{n-1} D(c x)-D\left(c^{n-1}\right) x^{n}-(n-1) D\left(c^{n-2} x^{n}\right) c \\
& -c^{n-1} D\left(x^{n-1}\right) x-(n-1) c^{n-2} x^{n} D(c)
\end{aligned}
$$

for all $x \in R$. Setting $x_{1}=x$ and $x_{2}=x_{3}=\cdots=x_{n-1}=c$ in the complete linearization of (65) we get

$$
\begin{gathered}
-(n-1) D\left(c^{2 n-3} x\right)+(n-2) c^{2 n-4} D(c) x+(n-1) D\left(c^{2 n-4} x\right) c \\
+c^{2 n-4} D(c x)+(n-2) c^{2 n-4} x D(c)-c^{n-1} D\left(x c^{n-2}\right)=0
\end{gathered}
$$

for all $x \in R$. Then substituting $x$ for $c x$ in relation (66) we obtain

$$
\begin{gathered}
-(n-1) D\left(c^{2 n-2} x\right)+(n-2) c^{2 n-3} D(c) x+(n-1) D\left(c^{2 n-3} x\right) c \\
\quad+c^{2 n-4} D\left(c^{2} x\right)+(n-2) c^{2 n-3} x D(c)-c^{n-1} D\left(x c^{n-3}\right)=0
\end{gathered}
$$

for all $x \in R$. Multiplying identity (66) by $c$ we get

$$
\begin{gathered}
-(n-1) D\left(c^{2 n-3} x\right) c+(n-2) c^{2 n-3} D(c) x+(n-1) D\left(c^{2 n-4} x\right) c^{2} \\
\quad+c^{2 n-3} D(c x)+(n-2) c^{2 n-3} x D(c)-c^{n} D\left(x c^{n-2}\right)=0
\end{gathered}
$$

Comparing the last two identities, we have

$$
\begin{aligned}
& -(n-1) D\left(c^{2 n-2} x\right)+2(n-1) D\left(c^{2 n-3} x\right) c \\
& \quad+D\left(c^{2} x\right) c^{2 n-4}-c^{n-1} D\left(c^{n-1} x\right)-(n-1) D\left(c^{2 n-4} x\right) c^{2} \\
& \quad-c^{2 n-3} D(x c)+c^{n} D\left(x c^{n-2}\right)=0
\end{aligned}
$$

for all $x \in R$. Substituting $x$ by $c x$ in (5) we get

$$
n D\left(c^{n} x^{n}\right)=n D\left(c^{n-1} x^{n-1}\right) c x+n c^{n-1} x^{n-1} D(c x)
$$

for all $x \in R$. Next, setting $x_{1}=x_{2}=\cdots=x_{n-1}=c$ and $x_{n}=c x^{n}$ in the complete linearization of (5) we have

$$
\begin{aligned}
n D\left(c^{n} x^{n}\right)= & D\left(c^{n-1}\right) c x^{n}+(n-1) c D\left(c^{n-1} x^{n}\right) \\
& +c^{n-1} D\left(c x^{n}\right)+(n-1) c^{n-1} x^{n} D(c) .
\end{aligned}
$$

Comparing the last two identities we see that

$$
\begin{aligned}
& D\left(c^{n-1}\right) x^{n}+(n-1) D\left(c^{n-1} x^{n}\right)+c^{n-2} D\left(c x^{n}\right) \\
& \quad+(n-1) c^{n-2} x^{n} D(c)-n D\left(c^{n-1} x^{n-1}\right) x-n c^{n-2} x^{n-1} D(c x)=0 .
\end{aligned}
$$

Setting $x_{1}=x_{2}=\cdots=x_{n-1}=c$ and $x_{n}=x$ in the complete linearization of (69) and using (68) we get

$$
\begin{aligned}
& D\left(c^{n-1}\right) c^{n-1} x+c^{n-2} D\left(c^{n} x\right)+(n-1) c^{2 n-3} x D(c)-D\left(c^{2 n-2}\right) x \\
& \quad+(n-1) D\left(c^{2 n-3} x\right) c-2 c^{2 n-3} D(c x)-(n-1) c^{2 n-4} x D\left(c^{2}\right)+c^{2 n-4} D\left(c^{2} x\right) \\
& \quad-c^{n-1} D\left(c^{n-1} x\right)-(n-1) D\left(c^{2 n-4} x\right) c^{2}+c^{n} D\left(x c^{n-2}\right)=0 .
\end{aligned}
$$


Using the last identity and (67) we obtain

$$
\begin{aligned}
& D\left(c^{n-1}\right) c^{n-1} x+c^{n-2} D\left(c^{n} x\right)+(2 n-3) x c^{2 n-3} D(c) \\
& \quad-D\left(c^{2 n-2}\right) x-c^{2 n-3} D(c x)-(n-1) c^{2 n-4} x D\left(c^{2}\right) \\
& \quad+c^{2 n-4} D\left(c^{2} x\right)-c^{n-1} D\left(c^{n-1} x\right)+(n-2) c^{2 n-3} D(c) x=0
\end{aligned}
$$

and hence

$$
D\left(c^{n} x\right)=c^{n-1} D(c) x+c^{n-1} x D(c)+c^{n-1} D(c x)-c^{n-2} D\left(c^{2} x\right)+c D\left(c^{n-1} x\right)
$$

for all $x \in R$. Setting $x_{1}=x_{2}=\cdots=x_{n-1}=c$ and $x_{n}=c x$ in (8) we arrive at

$n D\left(c^{n} x\right)=D\left(c^{n-1}\right) c x+(n-1) D\left(c^{n-1} x\right) c+c^{n-1} D(c x)+(n-1) c^{n-1} x D(c)$.

Comparing the last two identities we obtain

$$
\begin{aligned}
D\left(c^{n-1} x\right)= & -c^{n-2} D(c) x-c^{n-2} x D(c) \\
& -(n-1) c^{n-2} D(c x)+n c^{n-3} D\left(c^{2} x\right) .
\end{aligned}
$$

Putting (55) into last equation we get

$$
\begin{aligned}
2 D\left(c^{n-1} x\right)= & (n-1) c^{n-2} D(c) x \\
& +(n-1) c^{n-2} x D(c)+2 c^{n-1} D(x) .
\end{aligned}
$$

Using (64) and (70) we now get

(72) $2 D\left(c^{n-1} x\right)=(n-2) c^{n-2} D(c) x+(n-2) c^{n-2} x D(c)+2 c^{n-2} D(c x)$.

Substituting $x$ by $c x$ in the last equation we get

$$
2 D\left(c^{n} x\right)=(n-1) c^{n-1} D(c) x+(n-1) c^{n-1} x D(c)+2 c^{n-1} D(c x) .
$$

Next we set $x_{1}=x_{2}=\cdots=x_{n-1}=c$ and $x_{n}=x$ in (8). This yields

$2 n D\left(c^{n-1} x\right)=2 D\left(c^{n-1}\right) x+2(n-1) D\left(c^{n-2} x\right) c+2 c^{n-1} D(x)+2(n-1) c^{n-2} x D(c)$.

Comparing the last two equations we get

$$
\begin{aligned}
2(n-1) D\left(c^{n-2} x\right)= & \left(n^{2}-4 n+2\right) c^{n-3} D(c) x \\
& +\left(n^{2}-4 n+2\right) c^{n-3} x D(c) \\
& +2 n c^{n-3} D(c x)-2 c^{n-2} D(x) .
\end{aligned}
$$

Setting $x_{1}=x_{2}=x$ and $x_{3}=\cdots=x_{n}=c$ in (8) we have

$$
\begin{aligned}
n D\left(x^{2} c^{n-2}\right)= & (n-2) D\left(x^{2} c^{n-3}\right) c+2 D\left(x c^{n-2}\right) x \\
& +(n-2) x^{2} c^{n-3} D(c)+2 x c^{n-2} D(x) .
\end{aligned}
$$


Using the last equation and (73) we get

$$
\begin{aligned}
(n-1) n D\left(x^{2} c^{n-2}\right)= & (n-1)(n-2) D\left(x^{2} c^{n-3}\right) c+\left(n^{2}-4 n+2\right) c^{n-3} D(c) x^{2} \\
& +\left(n^{2}-4 n+2\right) c^{n-3} x D(c) x+2 n c^{n-3} D(c x) x \\
& -2 c^{n-2} D(x) x+(n-1)(n-2) x^{2} c^{n-3} D(c) \\
& +2(n-1) x c^{n-2} D(x) .
\end{aligned}
$$

Using (62) in the last equation we arrive at

$$
\begin{aligned}
(n-1) n D\left(x^{2} c^{n-2}\right)= & (n-1)(n-2) D\left(x^{2} c^{n-3}\right) c+\left(n^{2}-4 n+2\right) c^{n-3} D(c) x^{2} \\
& +\left(n^{2}-4 n+2\right) c^{n-3} x D(c) x+2 n c^{n-2} D(x) x \\
& +n c^{n-3} D(c) x^{2}+n c^{n-3} x D(c) x-2 c^{n-2} D(x) x \\
& +(n-1)(n-2) x^{2} c^{n-3} D(c)+2(n-1) x c^{n-2} D(x) .
\end{aligned}
$$

Therefore we have

$$
\begin{aligned}
n D\left(x^{2} c^{n-2}\right)= & (n-2) D\left(x^{2} c^{n-3}\right) c+(n-2) c^{n-3} D(c) x^{2} \\
& +(n-2) c^{n-3} x D(c) x+2 c^{n-2} D(x) x \\
& +(n-2) x^{2} c^{n-3} D(c)+2 x c^{n-2} D(x) .
\end{aligned}
$$

Setting $x_{1}=x_{2}=x, x_{3}=c^{2}$ and $x_{4}=\ldots=x_{n}=c$ in (8) we have

$$
\begin{aligned}
n D\left(x^{2} c^{n-1}\right)= & 2 D\left(x c^{n-1}\right) x+D\left(x^{2} c^{n-3}\right) c^{2}+(n-3) D\left(x^{2} c^{n-2}\right) c \\
& +2 x c^{n-1} D(x)+x^{2} c^{n-3} D\left(c^{2}\right)+(n-3) x^{2} c^{n-2} D(c) .
\end{aligned}
$$

Using (72) in the last equation we obtain

$$
\begin{aligned}
n D\left(x^{2} c^{n-1}\right)= & (n-1) c^{n-2} D(c) x^{2}+(n-1) c^{n-2} x D(c) x \\
& +D\left(x^{2} c^{n-3}\right) c^{2}+(n-3) D\left(x^{2} c^{n-2}\right) c+2 x c^{n-1} D(x) \\
& +(n-1) x^{2} c^{n-2} D(c)+2 c^{n-1} D(x) x .
\end{aligned}
$$

On the other hand setting $x_{1}=x^{2}$ and $x_{2}=x_{3}=\ldots=x_{n}=c$ in (8) we have

$$
\begin{aligned}
n D\left(x^{2} c^{n-1}\right)= & (n-1) D\left(x^{2} c^{n-2}\right) c+(n-1) c^{n-2} D(c) x^{2} \\
& +(n-1) x^{2} c^{n-2} D(c)+c^{n-1} D\left(x^{2}\right) .
\end{aligned}
$$

Comparing last two equations we get

$$
\begin{aligned}
2 D\left(x^{2} c^{n-2}\right)= & (n-1) c^{n-3} x D(c) x+D\left(x^{2} c^{n-3}\right) c \\
& +2 x c^{n-2} D(x)+2 c^{n-2} D(x) x-c^{n-2} D\left(x^{2}\right) .
\end{aligned}
$$

Now using (74) and (75) we have

$$
\begin{aligned}
(4-n) D\left(x^{2} c^{n-3}\right) c= & 2(n-2) c^{n-3} D(c) x^{2}+\left(-n^{2}+3 n-4\right) c^{n-3} x D(c) x \\
& +(4-2 n) c^{n-2} D(x) x+2(n-2) x^{2} c^{n-3} D(c) \\
& +(4-2 n) x c^{n-2} D(x)+n c^{n-2} D\left(x^{2}\right) .
\end{aligned}
$$


Substituting $x$ by $c x$ in the last equation we obtain

$$
\begin{aligned}
(4-n) D\left(x^{2} c^{n-1}\right) c= & (2 n-4) c^{n-1} D(c) x^{2}+\left(-n^{2}+3 n-4\right) c^{n-1} x D(c) x \\
& +2(2-n) c^{n-1} D(c x) x+(2 n-4) x^{2} c^{n-1} D(c) \\
& +2(2-n) x c^{n-1} D(c x)+n c^{n-2} D\left(c^{2} x^{2}\right) .
\end{aligned}
$$

Now using (62) in the last equation we get

$$
\begin{aligned}
(4-n) D\left(x^{2} c^{n-1}\right) c= & (n-2) c^{n-1} D(c) x^{2}+\left(-n^{2}+n\right) c^{n-1} x D(c) x \\
& +(4-2 n) c^{n} D(x) x+(4-2 n) c^{n} x D(x) \\
& +(n-2) x^{2} c^{n-1} D(c)+n c^{n-2} D\left(c^{2} x^{2}\right) .
\end{aligned}
$$

Setting $x^{2}$ instead of $x$ into (71) we arrive at

$$
2 D\left(c^{n-1} x^{2}\right)=(n-1) c^{n-2} D(c) x^{2}+(n-1) c^{n-2} x^{2} D(c)+2 c^{n-1} D\left(x^{2}\right) .
$$

Comparing the last equation and (76) leads to

$$
\begin{aligned}
0= & \left(-n^{2}+3 n\right) c^{n-1} D(c) x^{2}+\left(-n^{2}+3 n\right) c^{n-1} x^{2} D(c) \\
& +(8-2 n) c^{n} D\left(x^{2}\right)-2\left(-n^{2}+n\right) c^{n-1} x D(c) x \\
& -(8-4 n) c^{n} D(x) x-(8-4 n) c^{n} x D(x)-2 n c^{n-2} D\left(c^{2} x^{2}\right) .
\end{aligned}
$$

Setting $x^{2}$ instead of $x$ into (63) and using (77) leads to

$$
\begin{aligned}
(8-4 n) c D\left(x^{2}\right)= & (8-4 n) c D(x) x+(8-4 n) c x D(x) \\
& +\left(n^{2}-n\right) D(c) x^{2}+\left(n^{2}-n\right) x^{2} D(c) \\
& -2\left(n^{2}-n\right) x D(c) x .
\end{aligned}
$$

Last equation can be rewritten as

$$
\begin{aligned}
(8-4 n) c D\left(x^{2}\right)= & (8-4 n) c D(x) x+(8-4 n) c x D(x) \\
& -\left(n^{2}-n\right)[[D(c), x], x] .
\end{aligned}
$$

Multiplying the above relation by $c$ gives

$$
\begin{aligned}
(8-4 n) c^{2} D\left(x^{2}\right)= & (8-4 n) c^{2} D(x) x+(8-4 n) c^{2} x D(x) \\
& -\left(n^{2}-n\right) c[[D(c), x], x] .
\end{aligned}
$$

Putting $c^{2}$ for $c$ in the relation (78) leads to

$$
\begin{aligned}
(8-4 n) c^{2} D\left(x^{2}\right)= & (8-4 n) c^{2} D(x) x+(8-4 n) c^{2} x D(x) \\
& -\left(n^{2}-n\right)\left[\left[D\left(c^{2}\right), x\right], x\right] .
\end{aligned}
$$

and considering $D\left(c^{2}\right)=2 c D(c)$ implies

$$
\begin{aligned}
(8-4 n) c^{2} D\left(x^{2}\right)= & (8-4 n) c^{2} D(x) x+(8-4 n) c^{2} x D(x) \\
& -2\left(n^{2}-n\right) c[[D(c), x], x] .
\end{aligned}
$$


Comparing the last relation with (79) gives

$$
[[D(c), x], x]=0,
$$

which reduces the relation $(80)$ to

$$
D\left(x^{2}\right)=D(x) x+x D(x)
$$

for all $x \in R$. In other words, $D$ is a Jordan derivation. By Herstein theorem $D$ is a derivation. The proof in case we have the relation (6) goes through in a similar way and will be omitted. The proof of the theorem is complete.

\section{REFERENCES}

[1] K. I. Beidar, M. Brešar, M. A. Chebotar and W. S. Martindale 3rd, On Herstein's Lie map conjectures II, J. Algebra 238 (2001), 239-264.

[2] K. I. Beidar and Y. Fong, On additive isomorphisms of prime rings preserving polynomials, J. Algebra 217 (1999), 650-667.

[3] M. Brešar, Jordan derivations on semiprime rings, Proc. Amer. Math. Soc. 104 (1988), 1003-1006.

[4] M. Brešar, Jordan mappings of semiprime rings, J. Algebra 127 (1989), 218-228.

[5] M. Brešar, Functional identities: A survey, in: Algebra and its applications, Contemp. Math. 259, Amer. Math. Soc., Providence, 2000, 93-109.

[6] M. Brešar, M. Chebotar and W. S. Martindale 3rd, Functional identities, Birkhäuser Verlag, Basel, 2007.

[7] M. Brešar and J. Vukman, Jordan derivations on prime rings, Bull. Austral. Math. Soc. 37 (1988), 321-322.

[8] M. Brešar and J. Vukman, Jordan $(\theta, \phi)$-derivations, Glas. Mat. Ser. III 26(46) (1991), $13-17$.

[9] Cheng-Kai Liu and Wen-Kwei Shiue, Generalized Jordan triple $(\theta, \phi)$-derivations on semiprime rings, Taiwanese J. Math. 11 (2007), 1397-1406.

[10] J. M. Cusack, Jordan derivations on rings, Proc. Amer. Math. Soc. 53 (1975), 321-324.

[11] M. Fošner and N. Peršin, A result concerning derivations in prime rings, Glas. Mat. Ser. III 48(68) (2013), 67-79.

[12] I. N. Herstein, Jordan derivations of prime rings, Proc. Amer. Math. Soc. 8 (1957), 1104-1110.

[13] L. Rowen, Some results on the center of a ring with polynomial identity, Bull. Amer. Math. Soc. 79 (1973), 219-223.

[14] J. Vukman, On derivations of standard operator algebras and semisimple $H^{*}$-algebras, Studia Sci. Math. Hungar. 44 (2007), 57-63.

[15] J. Vukman, Some remarks on derivations in semiprime rings and standard operator algebras, Glas. Mat. Ser. III 46(66) (2011), 43-48.

[16] J. Vukman, I. Kosi-Ulbl and D. Eremita, On certain equations in rings, Bull. Austral. Math. Soc. 71 (2005), 53-60.

M. Fošner

Faculty of logistics

University of Maribor

Mariborska cesta 7, 3000 Celje

Slovenia

E-mail: maja.fosner@um.si 
B. Majcen

Faculty of logistics

University of Maribor

Mariborska cesta 7, 3000 Celje

Slovenia

E-mail: benjamin.marcen@um.si

J. Vukman

Institute of mathematics, physics and mechanics

Jadranska 19, 1000 Ljubljana

Slovenia

E-mail: joso.vukman@guest.um.si

Received: 18.1.2017.

Revised: 13.11.2017. 